FORMATION Formation emploi

Revue française de sciences sociales

139 | Juillet-Septembre 2017

De l'autonomie dans les parcours professionnels

\title{
De l'autonomie en formation : enjeux et réalités chez les stagiaires de l'AFPA
}

Goals and reality of autonomy among adults training's public

Autonomie in der Ausbildung: Herausforderungen und Realitäten bei AFPA-

Praktikanten

De la autonomía en la formación : desafíos y realidades entre los pasantes de la AFPA

\section{Benjamin Saccomanno}

\section{OpenEdition}

Journals

Édition électronique

URL : http://journals.openedition.org/formationemploi/5182

DOI : 10.4000/formationemploi.5182

ISSN : 2107-0946

Éditeur

La Documentation française

Édition imprimée

Date de publication : 15 octobre 2017

Pagination : $51-70$

ISSN : 0759-6340

Référence électronique

Benjamin Saccomanno, «De l'autonomie en formation : enjeux et réalités chez les stagiaires de l'AFPA », Formation emploi [En ligne], 139 | Juillet-Septembre 2017, mis en ligne le 15 octobre 2017, consulté le 30 octobre 2020. URL : http://journals.openedition.org/formationemploi/5182 ; DOI : https:// doi.org/10.4000/formationemploi.5182 


\title{
De l'autonomie en formation : enjeux et réalités chez les stagiaires de l'AFPA
}

\author{
Benjamin Saccomanno \\ Sociologue, post-doctorant au Céreq et membre du CERTOP (Centre d'étude et de recherche \\ Travail Organisation Pouvoir), Toulouse, université Toulouse Jean Jaurès
}

Résumé

De l'autonomie en formation : enjeux et réalités chez les stagiaires de l'AFPA

Enjeu de nombreux dispositifs d'accompagnement vers l'emploi, l'autonomie demande à être interrogée empiriquement. À partir d'une enquête à l'AFPA (Association nationale pour la formation professionnelle des adultes), l'article décline trois formes d'autonomie révélatrices de parcours aux dynamiques différenciées. Si l'autonomie apparaît comme une ressource à maintenir ou un enjeu de conquête pour progresser, elle peut aussi cristalliser des tensions lorsque les relations sociales en stigmatisent le manque et que l'individu s'identifie par sa distance aux normes sociales. À partir de cas typiques, l'autonomie révèle ici, notamment, les formes de convergence, mais aussi de désaccords avec les acteurs de la formation.

Mots clés : formation des adultes; cheminement professionnel ; étude de cas ; compétence ; orientation tout au long de la vie

Abstract

Goals and reality of autonomy among adults training's public

Goal of many employment support procedures, autonomy needs to be questioned empirically. Based on a research inside the National Association for Adult Vocational Training (AFPA), the article describes three forms of autonomy that reveal paths to differentiated dynamics. If it appears as a resource to be maintained or a stake in conquest to progress, it can also crystallize tensions when social relations stigmatize its lack and lead the individual to identify himself through his distance from social norms. With the help of typical cases, autonomy reveals forms of convergence but also disagreements with the actors of the formation.

Keywords: adult training ; occupational paths ; case study ; skill ; lifelong guidance Journal of Economic Literature: M 53 ; J 24

Traduction : Auteur. 
L'autonomie représente la capacité d'un individu ou d'un groupe à agir et à se gouverner selon ses propres normes et règles qu'il estime adaptées au contexte. Si elle est capacité de production normative, elle est également liberté d'utiliser des règles, normes et conventions hétéronomes (Terssac, 1992 ; Terssac et Maggi, 1996).

L'étude de l'autonomie révèle en effet les cadres de perception des enjeux, contraintes et ressources à partir desquels les individus ou les collectifs agissent, décident et collaborent. L'analyse vise alors à comprendre, d'une part, sa dynamique, c'est-à-dire comment l'autonomie se maintient, se conquiert, voire se reconquiert et, d'autre part, quels sont les effets concrets des expériences sociales sur cette dynamique.

L'article mobilise cette conception de l'autonomie par les règles et sa dynamique à propos de la gestion des parcours professionnels. En effet, du côté des individus, l'autonomie représentera la capacité à orienter le cours de leur devenir vers des positions souhaitées. Avec Bénédicte Zimmermann (2011), la notion de parcours met l'accent sur la diversité des formes d'enchaînement de situations professionnelles au cours de l'existence, diversité corrélative à la sortie du modèle fordiste et au renforcement d'un système d'emploi "grippé » qui contribuent à une parcellisation des parcours (Caillaud et Zimmermann, 2011).

L'autonomie éclaire ici les appuis des compétences, du capital social et les effets structurels sur la construction des parcours afin que, séquence après séquence, ces derniers demeurent, pour les individus, dans un espace d'acceptabilité, lui-même sujet à variation. Reconnaître une pluralité de formes de parcours questionne la pertinence d'une conception uniforme de l'autonomie du point de vue des individus. Son maintien ou son renforcement, par exemple, revêtent-ils la même signification lorsque les parcours different ?

Le concept d'autonomie contrôlée, développé par Béatrice Appay (2005), conduit à répondre par la négative. En effet, l'autonomie ne prend pas la même forme selon les niveaux de qualification et la relation d'emploi. Pour Appay, en situation de faibles ressources mobilisables, l'autonomie prend les traits d'une capacité d'action bien moins empreinte de liberté que de limitation par des régulations hétéronomes. Interroger les conceptions de l'autonomie dans les parcours ne peut donc faire l'économie de l'antériorité des situations vécues, mais donc aussi des effets des systèmes organisationnels et relationnels enquêtés.

À cet effet, nous travaillons sur un corpus de données toutes issues d'une séquence similaire dans les parcours des enquêtés : 44 entretiens auprès d'adultes engagés volontairement dans des formations qualifiantes à l'AFPA', dans les secteurs de l'industrie, de l'informatique et du bâtiment. L'approche biographique utilisée nous a permis de reconstituer les parcours antérieurs et les logiques prévalant dans les engagements des

1. Association nationale pour la formation professionnelle des adultes. 
individus. Notre matériau nous permet ainsi de formuler l'hypothèse d'une pluralité de conceptions de l'autonomie saisissables à partir des parcours tels que se les représentent les personnes. La validation empirique de cette hypothèse nous conduit à interroger, d'une part, les déterminants de ces conceptions de l'autonomie que nous allons situer dans l'articulation entre le vécu du parcours et l'orientation souhaitée pour celui-ci, à l'issue de la formation et, d'autre part, les effets sur l'engagement en formation et la coordination avec les enjeux du système de formation.

Après avoir situé, dans un premier temps, les régulations encadrant la gestion individuelle des parcours, l'objet des trois points suivants sera de rendre compte d'une pluralité de conceptions de l'autonomie visée ; celles-ci seront appréhendées à partir des normes et règles de gestion des parcours mobilisées pour élaborer le projet de formation. L'analyse abordera également la rencontre avec l'action des opérateurs ${ }^{2}$.

Le dernier temps interrogera les effets différenciés de ces interactions et du passage en formation, selon ces conceptions observées durant la séquence étudiée. Nous nous réfèrerons, entre autres, à une deuxième vague d'entretiens menée deux années plus tard auprès des mêmes stagiaires.

Ceci appuiera notre hypothèse principale selon laquelle la coopération avec les acteurs du système de formation dépendrait de la convergence en matière de règles de gestion des parcours. Il faut en effet considérer que du côté des différentes institutions du système de formation, la question de l'autonomie dans les parcours s'envisage en termes d'employabilité $^{3}$ et de responsabilisation de la population active quant à la gestion de celle-ci.

L'intérêt d'une analyse à partir des parcours des destinataires est alors de dépasser le discours général qui veut que l'autonomie représente un idéal à la portée légitime de quiconque aspire à une forme de réalisation personnelle (Ehrenberg, 2010). Sans avoir l'ambition de restituer toutes les formes d'autonomie chez des stagiaires de la formation pour adultes, notre objectif est de mettre en lumière que l'effet formation

2. Outre les entretiens auprès des stagiaires, nous avons également réalisé 50 entretiens qui nous ont permis de reconstituer la chaîne de décisions et d'actions menant de l'élaboration (élus, chargés de mission...) à la mise en œuvre des programmes de formation (formateurs, responsables de centre...), ainsi que de l'adressage des candidats (conseillers emploi, psychologues) jusqu'à l'issue de leur formation (jurys). Ces données ne seront utilisées qu’à titre complémentaire.

3. La notion de "parcours " a progressivement été établie comme référence dans les lois régulant le marché du travail. Dans les accords nationaux interprofessionnels (ANI) du 20 septembre et du 5 décembre 2003, elle apparaît ainsi cinq fois à travers l'expression de "parcours de formation » qui doivent être "individualisés » ou "personnalisés ». Le temps long et la dimension parcellaire des parcours vont dans le sens de la nouvelle échelle temporelle " tout au long de la vie " dans l'accès à la formation. Avec les textes ultérieurs, ces notions forment une rhétorique autour de l'enjeu de "sécurisation des parcours " qui passe par le déploiement de dispositifs visant le "maintien et l'amélioration de l'employabilité» (Accord national interprofessionnel du 11 janvier 2008 sur la modernisation du marché du travail) en situation d'emploi mais aussi lors des séquences transitoires. Cf. Saccomanno, 2013, pp. 151-162. 
sur les parcours est préalablement cadré par la normativité des règles institutionnelles en matière de gestion des parcours. L'effet sera alors positif si les stagiaires font déjà montre d'une autonomie tournée vers l'accroissement de leur employabilité selon les exigences du marché du travail.

Pour cela, nous emploierons la méthode des cas (Zimmermann, op. cit. ; Corteel et Zimmermann, 2007), c'est-à-dire le recours à des parcours typiques éclairant à la fois les contextes économiques vécus, le rapport au marché du travail expérimenté et les différentes ressources grâce auxquelles les individus reconnaissent et ordonnent les possibilités qui s'offrent à eux en matière de construction de leur parcours. Les cas retenus pour cet article sont parmi les plus illustratifs de notre matériau. En effet, chacun présente une version quasi idéal-typique des trois dimensions dont la configuration révèle une forme particulière d'autonomie. Les cas ont ainsi été élaborés selon les articulations entre les enjeux relatifs à l'autonomie dans :

- les situations d'emploi connues depuis l'entrée sur le marché du travail ;

- la maîtrise du parcours professionnel exprimée notamment lors des transitions d'une séquence à une autre ;

- l'effet escompté du passage en formation du point de vue des stratégies identitaires ou de carrières déployées.

Que ce soit le parcours antérieur, les modalités transitionnelles entre les séquences le composant ou l'avenir escompté, l'autonomie intervient chaque fois, soit comme un enjeu à maintenir ou développer pour étendre l'espace des possibles à l'aide de ressources ainsi éclairées, soit comme une contrainte pesant sur des choix qui reflètent alors plutôt la faible capacité de production normative des personnes eu égard à leur position dans l'espace social et/ou à la conjonction de pesanteurs exogènes à leur situation professionnelle (par exemple la maladie ou la charge économique de proches).

Chaque cas explicité se trouve en formation au moment de l'enquête, dans une perspective de développement de soi qui lui est propre, c'est-à-dire rationalisée à partir de son parcours et des conditions structurelles qui ont encadré celui-ci. Nous allons donc réfléchir à ce que nous révèlent les conceptions de l'autonomie relativement à la construction des enjeux ayant guidé ces adultes vers la formation. Nous pourrons ensuite questionner le déroulement de l'accompagnement durant la formation et les effets de celui-ci a posteriori. 


\section{Encadré 1 : Constitution et morphologie du corpus}

Le corpus de 44 adultes a été constitué de juin 2008 à septembre 2009, dans le cadre d'une recherche sur la co-construction des ambitions d'adultes en formation. L'enquête reposait sur une proposition à la direction de l'AFPA (Association nationale pour la formation professionnelle des adultes), en dehors de toute commande. Un accord a permis une liberté de circulation et de prise de contact avec les formateurs et stagiaires. En contrepartie, ont été intégrées les sections de maintenance industrielle dont le remplissage était insuffisant pour la direction. L'accès libre au terrain n'assurait pas l'accès aux données, aucune obligation n'étant adressée aux stagiaires. L'appui de la direction consista à me mettre en contact avec deux formateurs en maintenance. $\mathrm{Ma}$ présence régulière dans les murs m'a alors permis d'entrer en contact avec d'autres formateurs et, aussi parfois, avec des stagiaires de l'industrie (automatique et informatique industrielle), du bâtiment (Technicien d'études bâtiment en dessin de projet, Technicien supérieur bâtiment en économie de la construction), du tertiaire de service en informatique (Concepteur Développeur en Système Électronique, Concepteur Développeur en Informatique, Technicien supérieur en gestion de réseau informatique) et du social (Conseiller en insertion professionnelle).

J'ai progressivement développé des contacts et convaincu des premiers "volontaires », et ainsi gagné la confiance d'autres. Les présentations collectives de l'enquête, puis les échanges individuels ont permis de lever le doute quant à une assimilation de la recherche à une commande de I'AFPA. La règle d'anonymat aida ensuite à réduire les craintes de certains indécis. Au final, la plus grande contrainte a été liée à l'emploi du temps de ces adultes, engagés 35 heures par semaine, auxquelles s'ajoutent le travail personnel et les responsabilités d'ordre privée (certains travaillant par ailleurs le week-end). Les périodes de stage voyaient une part conséquente des effectifs quitter la région durant plusieurs semaines. Le dépassement de ces contraintes répond en outre à la possibilité d'un biais selon lequel le corpus ne serait composé que des stagiaires les plus « coopératifs». Le recueil s'est ainsi fait entre opportunités et souci de construire un corpus reflétant la morphologie de la population des stagiaires AFPA dans la région de l'enquête $\left(^{*}\right)$. Les entretiens ont une durée moyenne de 2 heures et ont été retranscrits intégralement.

Concernant les profils des adultes interrogés, 42 étaient demandeurs d'emploi (répartis de façon équilibrée entre une mise au chômage "stratégique » pour financer le projet de formation ; une sortie de période alternant contrats courts et chômage ; et une situation de chômage "subi ») et deux bénéficiaient d'un congé individuel de formation. En termes de diplômes, la moitié possède un niveau Bac, général (quatorze) ou technologique (huit). Près d'un tiers sont diplômés du supérieur (quatre à $\mathrm{Bac}+2$; quatre à $\mathrm{Bac}+3 ;$ six à $\mathrm{Bac}+5$ et au-delà). Moins d'un cinquième possèdent un niveau inférieur au Bac (quatre sans diplôme, deux avec un CAP et deux avec un BEP - respectivement certificat d'aptitude professionnelle et brevet d'études professionnelles). Les diplômés d'un niveau Bac se répartissent plus équitablement entre les filières, avec néanmoins une majorité dans l'industrie, puis de façon à peu près équivalente entre le tertiaire de service et le bâtiment. Quant aux diplômés du supérieur, plus de la moitié se positionnent dans des formations du tertiaire.

$\left.{ }^{*}\right)$ : Parmi l'ensemble des stagiaires AFPA en Midi-Pyrénées, on trouve une forte majorité d'hommes (70 \% en $2009 ; 76 \%$ en 2010). Le faible nombre de femmes est à imputer aux filières accessibles à notre investigation. Le domaine des techniques du tertiaire concentre la plus forte part de femmes, mais nous n'avons pu y avoir accès. Toutefois, la majorité des formations de techniques du tertiaire relève des niveaux IV (Bac) et $V$ (BEP-CAP), regroupant des niveaux de qualification initiaux présentant une hétérogénéité moindre. C'est aussi dans ces sections que l'on observe les plus fortes probabilités de changements d'orientation en cours d'accompagnement, voire d'abandons de projets (Sources : Rapports annuels d'activité 2009, 2010, 2011 de l'AFPA). 


\section{Prescriptions institutionnelles : une gestion « entrepreneuriale » de l'autonomie?}

Comment mesurer l'autonomie des individus ? Celle-ci serait-elle obtenue légitimement lorsque l'on arrive au terme d'un accompagnement financé dans le but de renforcer l'autonomie des destinataires ? Telle qu'elle est présentée dans les référentiels et les documents programmatiques des politiques publiques de l'emploi, l'autonomie est-elle vraiment en phase avec la réalité des parcours individuels ? Les enjeux qu'elle révèle chez les individus sont-ils en convergence avec les normativités en présence au niveau de l'ensemble du système de formation ?

Dans le champ de l'insertion sociale, Nicolas Duvoux (2009) a livré une fine description des tensions et accommodements entre perceptions des usagers et exigences institutionnelles autour de l'enjeu d'autonomie. Il s'agit d'emprunter cette voie à propos des parcours professionnels : les trois formes d'autonomie signalées se distinguent, entre autres, par la fluidité ou, au contraire, les percussions des rapports entre stagiaires et opérateurs des politiques de l'emploi. Pour mieux saisir cela, il convient de synthétiser les prescriptions institutionnelles relatives à l'autonomie dans la gestion des parcours.

Le développement de l'autonomie passe, notamment, par la maîtrise et la mise à jour régulière des savoirs et compétences, au gré des innovations techniques et économiques (Auer et Gazier, 2010). Elle se donne à voir comme un objet à sécuriser : si elle constitue une préoccupation des programmes d'intermédiation publique vers l'emploi, c'est avant tout du point de vue d'un degré minimal à assurer témoignant d'un niveau d'employabilité théoriquement suffisant pour maintenir les chances de trouver du travail (Larquier et Remillon, 2008). Comme la capacité de mobilisation et l'adhésion aux impératifs institutionnels, l'autonomie est un repère d'ordonnancement des destinataires des politiques d'insertion selon leur degré d'accointance à un " habitus entrepreneurial " inculqué par les agents de l'action publique (Mauger, 2001). Les propos ci-dessous, issus d'un entretien avec un acteur à l'articulation des producteurs et des opérateurs des programmes résument cela :

"On ne perd jamais de vue que notre objectif, c'est de donner un minimum de compétences suffisantes pour que le demandeur d'emploi puisse intégrer une entreprise à l'issue de l'action. Je pèse mes mots quand je dis ça, parce que nous n'avons pas la prétention, quand on nous confie une personne qui n'a aucune connaissance d'un métier, de la rendre productive, mais employable. " (Chargé de mission formation professionnelle et apprentissage, Conseil Régional)

Cet extrait illustre aussi le poids des régulations institutionnelles sur la mise en œuvre des politiques de l'emploi, lesquelles, selon Emmanuelle Reynaud (2003), se stabilisent entre prescriptions européennes d'un traitement préventif de la dégradation de l'employabilité et déploiement national de dispositifs d'accompagnement resserrés à 
vocation curative de l'éloignement du marché. L'autre particularité des régulations des politiques de l'emploi et de la formation, relevée par l'auteure, est également de laisser une " marge d'interprétation et de jugement à l'intérieur même des organisations hiérarchisées", ce qui peut renforcer l'autonomie des opérateurs dont l'élaboration et la mise en œuvre des actions sont largement structurées par l'état des marchés locaux de l'emploi sur leur territoire. (Saccomanno, 2011).

Face à ces prescriptions officielles des règles de «bonne gestion » des parcours, les destinataires font parfois l'expérience d'un décalage cognitif important en début d'accompagnement, tant leur propre répertoire pour décrire leur cheminement et expliciter les raisons de leurs orientations passées et présente en est éloigné. Ce décalage s'avère plus ou moins problématique selon les modes individuels de gestion des parcours et les réalités économiques affrontées. Ainsi, si les trois cas représentent chacun une génération différente, ils vont aussi se distinguer par leur degré de proximité avec l'habitus entrepreneurial mentionné plus haut par Mauger (op. cit). Notre sélection de ces cas s'explique par l'hétérogénéité des expériences du marché du travail et du rapport à la carrière qu'ils expriment. Les conditions d'entrée sur le marché, puis les différentes situations et positions connues vont ainsi permettre de comprendre les conceptions différenciées de l'autonomie qui ont guidé Jean-Jacques, Samy et Laetitia vers l'AFPA.

\section{L'autonomie maintenue comme ressource de parcours gérés étape par étape}

À 55 ans, Jean-Jacques entre dans la catégorie des séniors. S'il suit une formation de Technicien supérieur en automatique et informatique industrielle (TSAII, niveau $\left.\mathrm{III}^{4}\right)$, c'est dans une optique de capitalisation et de mise à jour des savoirs retirés de ses multiples expériences en tant qu'électro-technicien. Il a en effet l'opportunité de les mobiliser comme technicien formateur au service d'un groupement d'employeurs pour lequel il travaille de façon ponctuelle depuis plusieurs années, et avec lequel il a négocié la création d'un poste à sa sortie de formation, elle-même financée par ses droits sociaux. Il présente ce poste comme la synthèse de son parcours, débuté comme technicien en électricité et électronique, puis évoluant entre informatique, enseignement technique vacataire ou encore technicien de maintenance. Cette synthèse parachève un parcours retenu ici car l'autonomie y apparaît, d'une part, comme le produit de ressources capitalisées et d'opportunités saisies et, d'autre part, comme une capacité à maintenir afin de poursuivre une stratégie d'adaptation des positions professionnelles aux situations personnelles.

4. Le niveau inscrit correspond à celui du titre professionnel qu'obtiendront les stagiaires validant leur formation, soit ici deux années après le baccalauréat. 


\subsection{Les bénéfices actuels d'un marché du travail autrefois ouvert}

Jean-Jacques situe son entrée sur le marché du travail à l'âge de quinze ans. Il explique cette première séquence par l'héritage familial, mais aussi en réaction à la situation économique de son foyer. Deuxième d'une lignée de sept enfants d'une famille monoparentale modeste, il suit à 13 ans les pas de son frère aîné lorsqu'il s'engage à l'école nationale de la marine marchande de Saint-Malo. Ce choix de secteur n'est pas hasardeux : petit fils de marin, c'est aussi pour lui l'occasion de s'immerger dans un univers culturel et professionnel qui l'attire depuis son plus jeune âge. Sa capacité de choix est renforcée par l'agrément de sa mère qui, un an et demi après son entrée dans cette école, abondera encore dans son sens lorsqu'il décide d'arrêter :

" J'étais à l'école d'apprentissage maritime pour avoir un CAP d'électromécanicien et je me plaisais plus à naviguer sur les bateaux qu'à bûcher, donc je suis parti naviguer (...) Ça me plaisait pas, donc ma mère a dit "C'est pas la peine que je paye, autant que tu repartes naviguer, au moins tu gagneras des sous et tu verras ce qui te plaît". Plus on travaillait tôt plus ça lui convenait ; mais comme c'était ce qu'on recherchait, ça nous convenait bien quelque part."

Entré sur le marché du travail à la fin des années 1960 sans diplôme, Jean-Jacques obtient un équivalent CAP d'électricien à l'AFPA, en 1973. Il fait partie de ces derniers arrivants avant que le système d'emploi ne se grippe. Sa faible qualification n'était pas un frein dans un contexte de plein emploi ; il la compensait par les expériences qu'il a pu accumuler dans le secteur de la marine marchande dès son entrée sur le marché du travail à 16 ans en 1969. Ce secteur, à l'instar de l'industrie à la même époque, bénéficiait d'une conjoncture d'emploi favorisée par l'intensification des activités économiques et commerciales (Cassagnou, 2003). Son titre d'électricien lui assure en effet de travailler comme électromécanicien naviguant jusqu’à la fin des années 1970, avant de décider de s'installer et de fonder un foyer. En 1983, après avoir patienté deux années sur liste d'attente, il suivra une nouvelle formation qualifiante, dans un domaine balbutiant, mais prometteur à l'époque : l'informatique appliquée à l'automatisme industriel. Son titre obtenu, il occupera différentes fonctions dans la même entreprise jusqu'en 1999.

Le parcours de Jean-Jacques est ainsi mis en récit sous une apparente fluidité, bien que l'articulation de certaines séquences nécessite parfois d'occuper des postes éloignés de son cœur de métier, ce qu'il accepte notamment lorsqu'il patiente avant d'intégrer sa deuxième formation. Ses décisions d'orientation ne sont pas prises par défaut, mais découlent d'arbitrages opérés suite à des opportunités qui s'offrent à lui. Son parcours se révèle bien moins planifié à partir d'un modèle de carrière typique que construit par adjonction de séquences produites par les évènements et opportunités qui reconfigurent ainsi l'espace des possibles à l'intérieur duquel il prend ses décisions (Longo et al., 2013). 


\subsection{L'autonomie comme maintien d'un espace ouvert des possibles}

L'autonomie apparaît dans les décisions et les changements de situation visés. Elle est tout d'abord une capacité à réagir à un contexte pouvant se reconfigurer à la faveur des dynamiques du marché du travail ou personnelles. Elle repose ensuite sur une réserve de compétences dont la valorisation initiale dans le système d'emploi renforce une capitalisation des savoirs.

Cette capitalisation bénéficie par ailleurs d'un rapport non conflictuel aux institutions et intermédiaires du marché du travail. Comme d'autres stagiaires d'âge approchant interrogés, Jean-Jacques sollicite plusieurs fois dans son parcours des dispositifs publics de formation pour se mettre à jour de nouvelles technologies et enrichir son CV. Ses décisions révèlent une stratégie de maintien de sa capacité d'orientation de son parcours par l'actualisation régulière de ses savoirs ce qui, sans que cela ne soit formalisé ainsi de son point de vue, converge avec les prescriptions institutionnelles.

Cette convergence révèle en effet, de son côté, la stabilisation d'une règle d'ajustement des savoirs au contexte d'emploi qui lui semble correspondre au sens souhaité pour son parcours. Cette règle se traduit par une réflexion préalable en termes d'ouverture des possibles professionnels, au regard de l'état des marchés internes ou externes tels qu'il se les représente. Il n'interagira que très peu avec les agents du service public de l'emploi, car il ne les sollicite qu'une fois son projet de formation déjà arrêté.

Il gère ainsi son employabilité par acquisition de compétences, mais aussi par le déplacement vers des espaces qui lui permettront de valoriser son capital. C'est ce qu'il fera entre 1983 et 1999 , lorsqu'il se reconvertira comme formateur dans son entreprise. S’il n'a jamais eu d'expérience en la matière, c'est aussi pour lui l'opportunité de relancer son engagement professionnel, tout en anticipant une moindre pénibilité physique dans les années à venir :

"Y'avait d'autres techniciens qui venaient, qu'il fallait qu'on commence à former pour pouvoir prendre la relève et regrouper par secteur. Donc ils cherchaient à ce moment-là un formateur. J'avais été voir le DRH pour savoir, il me dit 'Pourquoi t'irais pas voir là-bas? Ils sont en train de chercher un formateur, peut-être que tu peux le faire. Va postuler'. Donc j'écris ma lettre sur le bureau, tac, ils transmettent, tout de suite derrière j'ai été convoquévu que c'était dans la même enceinte (rire). Voilà, ils m'ont dit 'Demain tu viens et puis tu leur montres ce que tu sais faire'. Donc, j'ai été là-bas, le lendemain j'ai commencé mon premier cours et ça m'a plu. Ma prestation leur a plu aussi, donc j'ai été embauché sur le poste et là j'ai fait 7 ans. "

Cette séquence se terminera par un licenciement économique lorsque son entreprise fusionnera avec une autre. Dès lors, il peut décider de poursuivre sa reconversion vers l'enseignement ; il s'y essaiera durant plusieurs années, sans toutefois dépasser le statut de professeur remplaçant. Si la stabilité statutaire et économique de sa compagne amortit un temps l'incertitude que ces années nourriront vis-à-vis de son avenir 
d'enseignant en lycée professionnel, l'argument économique du foyer le conduira à reprendre temporairement une activité de technicien sur des grands équipements. Mais un accident du travail rétrécira l'espace des possibles qu'il envisage : ses choix d'orientation plus contraints révèleront un écart trop important entre ses compétences et ce que requièrent les postes visés. Sa formation au moment de l'enquête reprend le même processus que lors de sa bifurcation : entré comme technicien, ses savoirs, ainsi que désormais ses expériences dans la formation, rencontreront les besoins de son employeur.

Avec ce premier cas, l'autonomie dans les parcours s'illustre dans le rapport aux positions jugées réalistes et acceptables. Assurée par des compétences rencontrant la plupart du temps les besoins des recruteurs, cette première autonomie apparaît finalement peu négociée avec d'autres que les proches ou soi-même : selon un registre d'équilibre économique d'une part, d'adaptation des ambitions aux dynamiques personnelles et du marché, de l'autre. Le cas suivant démontre une autonomie bien plus contrôlée (Appay, 2005) au sens où les règles de gestion du parcours ne semblent que très peu appartenir au sujet.

\section{Des parcours éprouvants à l'autonomie négative?}

Le deuxième cas est celui de Samy, 28 ans lors de notre premier entretien durant sa formation de technicien en maintenance industrielle. Français dont les parents Tunisiens ont migré en France dans les années 1960, il met en récit un parcours typique de jeunes n'ayant eu, jusque-là, que peu de ressources à faire valoir face aux discours institutionnels et aux réalités économiques. Son parcours est au contraire marqué par des expériences scolaires et professionnelles stigmatisant son manque d'autonomie. Son parcours apparaît en effet constamment guidé par la menace de perdre le peu de prise qu'il semble avoir sur lui.

Nous convoquons ce cas pour sa représentativité de parcours qui dénotent une restriction progressive de l'espace reconnu des possibles. Il concerne des individus exprimant une dépossession grandissante de leurs capacités de choix et d'action, dynamique que le passage en formation est censé, sinon rompre, du moins atténuer.

L'autonomie renvoie ici à un principe de justice non accompli : sa mention met en cause les lacunes des institutions rencontrées qui n'ont pas réussi à fournir les ressources d'une insertion sociale et professionnelle. Au contact des représentants de l'école, recruteurs ou acteurs des politiques de l'emploi, ces individus ont connu des orientations non désirées et vu leur identité blessée par la violence de jugements qui ont entamé leur propre image et confiance en eux-mêmes (Martuccelli, 2006), comme nous allons le voir. Pour ces individus, admettre le besoin de recourir à un dispositif tel que la forma- 
tion professionnelle pour adultes présente en fait le risque de stigmatiser encore plus leur défaut d'autonomie, car les échanges les renvoient à leur dépendance institutionnelle. L'autonomie apparaît ainsi négative lorsque les relations institutionnelles sensées la développer ont finalement plus pour effet d'en stigmatiser les lacunes.

\subsection{Echec initial et contraintes majorées sur les parcours}

Catégorisé en échec par l'institution scolaire dès le secondaire, le cas de Samy démontre la corrélation avec une orientation contrariée repérée par Brinbaum et Guégnard (2012) chez les jeunes d'origine immigrée. Il cumule un faible niveau d'information sur les cursus existants et une absence de capitaux sociaux ou économiques compensatoires. Cela se traduit alors par une impossible construction de jugement qui intégrerait sa future employabilité.

Décidé à entrer rapidement sur le marché du travail, le seul critère qu'il convoque pour son orientation de fin de troisième est la part de pratique contenue dans les formations. Mais son entrée en BEP est d'abord un choix par défaut : poussé à s'orienter vers une filière technique par le conseil de classe, Samy dédaigne le CAP "par orgueil» dira-t-il, "parce qu'au collège, c'était mal vu le CAP : c'est la honte, tu fais CAP parce que t'es un mongol, c'est pour les cramés et tout". Son choix de filière électro-technique est ensuite le produit d'une confusion entre électricité et électronique : lui qui souhaitait apprendre "à travailler sur des télévisions, des ordinateurs, des trucs comme ça ", il se retrouve dans une formation qui le prépare à installer des systèmes électriques. Les cours en atelier lui sembleront insuffisants pour développer une aisance technique, ce qui forgera chez lui la sensation d'avoir fait le mauvais choix : "C'est vrai que je me disais que peut-être le CAP, le fait que c'est avec un patron, c'est peut-être ça que j'aurais du faire ".

Bloqué dans ce premier non-choix, la transition de la formation vers l'emploi sera difficile, car son faible niveau de qualification et son inexpérience lui barreront l'accès aux emplois stables comme aux contrats précaires sur lesquels il misait pour compenser son insuffisance technique :

"C'est un peu la barrière du débutant: tu postules et on te dit: 'Ok, vous avez le diplôme mais pas l'expérience'. Alors toi tu réponds: 'Oui, mais acceptez-moi pour que je puisse la faire'. Et là on te renvoie: 'Non. Mais revenez nous voir quand vous aurez de l'expérience' et ainsi de suite... Tu le sentais bien dans l'électricité ce truc-là, parce que franchement c'était des mecs compétents qu'ils voulaient, ils allaient pas prendre un mec qu'ils étaient pas sûrs... Donc, ouais, javais cette barrière-là, donc elle m'a encore plus refroidi de mon manque de confiance et tout ça. À tel point que je ressentais le besoin d'être formé, les souvenirs me remontent... même si ça a pris du temps avant de vraiment le faire. "

Cet extrait est particulièrement révélateur de l'expérience d'entrée sur le marché qui peut générer, chez des jeunes peu qualifiés, une conception menacée de l'autonomie. Tout d'abord, dans la lignée de son expérience scolaire, Samy se heurte à une succession 
d'évaluations négatives de ses capacités à occuper le poste pour lequel il a été formé. Le stade du refus pour manque d'expérience, qu'il ne parvient à dépasser, le condamne à rester dans un cercle vicieux et paradoxal de celui qui ne décroche jamais la première expérience dont l'absence lui est dans le même temps reprochée. Un rapport ambivalent va alors se développer à l'égard du marché du travail, entre rejet et intériorisation des règles de son fonctionnement.

Le fonctionnement du marché est critiqué du fait de ses normes de recrutement discriminantes que Samy ne tentera de contourner que dans l'espoir de mieux y souscrire. En effet, de l'obtention de son BEP à son entrée à l'AFPA, une séquence de dix années s'écoule, faite de petits boulots non qualifiés, travail au noir, CV truqués et projets avortés de retour en formation, car il se détourne chaque fois avant la fin du processus de candidature.

Cette séquence nous semble ainsi typique de parcours structurés par une autonomie contrôlée des sujets. Suivant le concept d'Appay (op. cit., p. 161) les sociétés démocratiques dites « avancées " verraient leurs membres, particulièrement les plus fragiles, s'orienter de façon apparemment libre, tout en les mettant dans des conditions d'impossibilité de choix, car les prescriptions de gestion des trajectoires n'engagent qu'à adopter, malgré soi ou de façon consentante, les solutions « responsables » qui renforceront leur flexibilité et leur performance. La fin de l'extrait ci-dessus démontre bien comment Samy intériorise les normes de recrutement mobilisées par les professionnels qui l'ont rejeté. Celles-ci se normalisent tout en renforçant chez lui l'idée que, bien que diplômé, il n'est pas encore à la hauteur de leurs attentes (" un mec pas sûr»), ce qui finira par justifier de retourner se former.

\subsection{L'autonomie vécue par défaut}

L'autonomie contrôlée du parcours de Samy devient un attribut stigmatisant de son identité sociale, dès lors que les échanges le renvoient à sa dépendance aux politiques sociales s'il souhaite s'insérer professionnellement. De ses interactions avec les acteurs des politiques de l'emploi, nous voyons apparaître une autonomie négative, c'est-à-dire vécue principalement par défaut, à la fois faible et menacée.

L’enjeu de cette autonomie négative est alors le rattrapage d'une normalité, par opposition à une conquête sociale et économique. Dans les parcours, l'autonomie négative nous semble donc indiquer un point de vue spécifique d'individus plongés dans des situations qui les renvoient à leur manque de capacité de choix, ainsi qu'à la faiblesse des ressources qu'ils mobilisent. Cette forme d'autonomie que nous repérons se vit donc à travers le risque de continuer à suivre la pente empruntée jusque-là par les parcours.

Ainsi, Samy épiloguera sur la pression sociale qu’il ressent à l'approche de ses trente ans, pression que nous pouvons lire en termes de crainte de se situer en dehors d'une norme projetée des réalisations légitimes selon les âges de la vie (mise en couple, logement, paren- 
talité... $)^{5}$. Face à cette crainte d'être en défaut par rapport à une norme sociale, Samy ne voit finalement qu'une ressource qui pourrait lui permettre d'avancer : bien qu'il ait qu'obtenu son BEP dix ans auparavant après une orientation par défaut, et malgré qu'il n'ait jamais réussi à en capitaliser les savoirs (il avouera se replonger régulièrement dans ses cours pour " garder le niveau »), son choix de filière répond à une logique d'amortissement du coût de l'engagement en ne repartant pas de zéro :

"J'ai encore des restes de mon diplôme, même si ça date d'il y a dix ans. Je l'ai toujours gardé à l'esprit, donc si je dois prétendre me reformer dans quoi que ce soit, faut quand même rester à peu près cohérent, sinon ces deux années d'études seraient perdues et ça, ça m’embêterait énormément de tout balancer."

Le cas de Samy est révélateur d'une forme d'autonomie dont l'expérience vécue est le produit de l'échec des séquences scolaires et d'entrée sur le marché du travail, échec attribué à la faiblesse des ressources mobilisables que l'individu ne parvient à compenser. Cette autonomie se vit en négatif car, si elle met en exergue le contrôle sur la gestion des parcours qu'exercent les normes et règles hétéronomes, les échanges avec des acteurs du marché renforcent une tension entre éloignement de ces normes et impossibilité de les contourner. Ainsi délestés de toute capacité de production normative quant à la gestion de leurs parcours, les individus n'en apparaissent que plus dépendants à l'égard des dispositifs publics. Le risque est alors de favoriser le développement de comportements retraduisant une certaine violence des jugements reçus à leur égard. Nous verrons justement dans la partie suivante que, pour Samy, le passage en formation reproduit le caractère éprouvant des relations institutionnelles connues jusque-là. Depuis sa sortie de formation initiale, aucun dispositif public d'accompagnement n'est parvenu à co-construire avec lui un projet de stabilisation professionnelle.

De fait, ce type de parcours interroge l'effet stigmatisant de l'échec des politiques scolaires et d'insertion qui génère, par la suite, une appréhension institutionnelle par défaut des individus, c'est-à-dire du point de vue de leurs seules difficultés à se mettre en conformité avec des normes sociales présentées comme légitimes (Delory-Momberger et Laniez, 2009).

\section{Du retrait à la conquête : I'autonomie renégociée}

Le dernier cas retenu est celui de Laetitia, 25 ans, engagée dans une formation de technicienne d'études dans le bâtiment, spécialisée en dessin de projet. Ce cas illustre de

5. Dans les représentations collectives, les âges apparaissent comme des constructions sociales qui organisent les conceptions individuelles des expériences et reproduisent ainsi une échelle de normalité exogène à celui qui s'y confronte (Elias, 1996). 
quelle manière l'autonomie dans les parcours met en relation les sphères professionnelle et privée.

Plus précisément, à l'encontre des discours institutionnels n'attachant les enjeux de l'autonomie qu'à des questions de carrières et de sécurisation des positions, le parcours de Laetitia démontre une conception de l'autonomie tournée vers un équilibre entre ses engagements au travail et ses relations personnelles. Les moments de reconfiguration de ces équilibres constituent des nœuds dans les parcours, c'est-à-dire des moments décisifs d'articulation entre deux séquence, et l'autonomie s'appréhende alors comme la capacité des individus à orchestrer ces rééquilibrages des engagements entre eux lorsque leur ambition se renégocie (Saccomanno, 2016).

\subsection{Le retrait stratégique des considérations de carrière}

Le parcours de Laetitia débute par un véritable retrait, d'abord des études, puis d'un projet de carrière professionnelle. En quelques mots, elle synthétise des motifs qui la rapprochent de l'identité de retrait perçue par Sainsaulieu (1985) chez les salariés les plus fragilisés statutairement :

"Après mon bac littéraire, que j'ai été surprise d'avoir, je suis partie à la fac d'anglais... même si j'étais très nulle en anglais. J'ai fait beaucoup de choses à part étudier.. Alors je me suis aperçue que j'étais allée en fac sans savoir quoi faire. Et c'est là que j'ai rencontré mon copain et je suis partie le rejoindre à la campagne. Donc il a fallu travailler, mais sans formation, je pouvais pas espérer de bons boulots, mais au départ je m'en foutais, c'était pas important. C'est vrai que tant que je ne m'étais pas stabilisée dans ma vie personnelle, je ne m'étais pas trop aperçue de l'importance de ma vie professionnelle."

Au travail, l'identité de retrait se caractérise, chez Sainsaulieu, par une expérience subie du pouvoir dans les rapports sociaux : les salariés pas ou très peu qualifiés concernés démontrent une absence de capacité de décision et des marges d'autonomie réduites uniquement à ce que leur permet le contremaître. Ce qui permet de tenir dans ces situations d'après Sainsaulieu est que l'activité est le moyen de soutenir la vie extra-professionnelle. Le cas de Laetitia nous permet d'approfondir les autres facettes de son identité qui, dans les premiers temps de son parcours, la positionne dans un retrait de toute responsabilité qu'on pourrait lui imposer contre son gré. En effet, la précarité statutaire des postes peu qualifiés qu'elle occupe entre ses 19 et ses 24 ans relève en fait d'une stratégie visant à conserver la maîtrise des impératifs professionnels sur sa vie privée. De son aveu, elle veille alors à limiter ses engagements contractuels afin de ne pas se sentir "piégée par un CDI» (contrat à durée indéterminée). Son cas démontre l'existence de parcours accommodés à une incertitude statutaire. Loin de vivre quotidiennement le risque de désaffiliation comme dans le cas précédent, le contexte du marché du travail et les interactions avec les employeurs n'entament pas l'évaluation que fait Laetitia de sa situation. L'autonomie dans les parcours n'apparaît donc pas réductible aux registres 
institutionnels d'évaluation de l'employabilité, dès lors que l'on s'intéresse à des parcours dont la dynamique provient d'une revendication à se construire en priorité dans le cadre de la sphère privée.

Par-delà d'apparents déterminismes structurels, l'autonomie peut ainsi révéler une stratégie de reconnaissance de son altérité face à des normes et règles de gestion des parcours. Jusqu'à son engagement volontaire à l'AFPA, les décisions de Laetitia expriment une liberté de choix exercée dans le refus de certaines assignations lors d'échanges avec des intermédiaires de l'emploi (principalement agences intérim et conseillers emploi) ou des fréquentations :

"Tout le monde me disait: 'pourquoi tu ne fais pas aide-soignante?', 'parce que je n'en ai pas envie', ça ils ont eu du mal à comprendre. En tant que filles quand on cherche du travail on nous propose dans le tertiaire ou les métiers du sanitaire et social... Donc, je suis allée m'intéresser à ce que l'on proposait aux garçons. (...) Et puis, le côté des rapports humains dans ces milieux-là m'intéressait plus, comme quand j'ai fait chauffeur livreur : j'étais dans une ambiance qui mallait super bien alors que je ne connaissais que dalle aux pièces automobiles, rien à voir avec quand je faisais des ménages à ne fréquenter que des aides-soignantes et les infirmières par exemple, alors que normalement, il paraît qu'une fille ferait mieux de faire ces métiers..."

\subsection{L'autonomie ou la liberté de s'engager vers de nouveaux espaces de reconnaissance}

La formation de technicienne dans le bâtiment qu'elle suit révèle d'autres enjeux que les seules questions qui, jusque-là, guidaient ses choix. D'une posture initialement distanciée face au monde du travail, elle en arrive à éprouver un besoin de réalisation croissant, sans pour autant parvenir à définir une activité professionnelle correspondante. Ce rapport au travail connaît alors un revirement lorsqu'elle prend connaissance du métier de dessinateur dans le bâtiment - "une illumination ", "une évidence " - générant un passage à l'action immédiat : "Autant j’ai passé des années à savoir que je voulais faire quelque chose, mais à faire un pas en avant, deux pas en arrière; autant le jour où j'ai connu cette formation, j'ai foncé à l'AFPA pour les tests!».

Cet engagement n'est donc pas un effet d'injonctions sociales de son entourage, ni de conseillers ; il marque une bifurcation produite par un événement non anticipé qui reconfigure ses propres normes de jugement en matière de gestion de son parcours professionnel. Dans le cas de Laetitia, cela est rendu possible grâce à l'assise de sa situation amoureuse : cela signifie, d'une part, une compatibilité matérielle des engagements et, d'autre part, que ces nouvelles normes restent cohérentes avec celles de sa sphère privée dans laquelle elle a trouvé jusque-là les conditions de sa propre reconnaissance auprès de ses proches avec qui elle partage des "représentations d'une vie réussie dans le cadre de la communauté" (Honneth, 2000, p. 155). 
Deux ans plus tard, son autonomie reste au service de ces représentations : après avoir travaillé comme dessinatrice depuis l'obtention de son titre, la carrière est de nouveau mise en suspens pour aborder la parentalité. Ce nouveau compromis s'appuie sur un accès désormais facilité à des postes qualifiés, mais il témoigne également d'une renégociation de l'autonomie comme ressource d'une stratégie de réalisation conjointe des projets familiaux et professionnels :

"On s'est dit que c'était le bon moment. (...) Mais je n'oublie pas le dessin, je vais y revenir. Et même avant de pouvoir bosser dans un cabinet ou autre, je vais chercher des contrats pour travailler depuis chez moi "

L'autonomie de Laetitia ne peut donc se penser en dehors de l'articulation entre les différentes sphères de sa vie sociale. De ce point de vue, elle apparaît bien moins limitée que si l'on s'en était tenu aux seuls déterminants objectifs des parcours que sont les niveaux de diplôme, d'expérience et de qualification des postes occupés.

Ce parcours nous semble ainsi typique de la montée des exigences de reconnaissance, de développement et d'épanouissement dans et en dehors du travail (Baudelot et Gollac, 2003), des exigences dont la résonnance varie au cours des existences et des structures relationnelles qui les encadrent.

L'autonomie est alors une ressource incontournable pour préciser et suivre ces exigences, même lorsqu'elles sont plus ou moins divergentes avec les discours institutionnels qui abordent les parcours de leur nécessaire sécurisation régulière, au risque, le cas échéant, de se mettre en position défavorable sur les plans sociaux et économiques. Notre dernier cas permet donc de relativiser cette rhétorique dont le propos ne peut s'avérer qu'incomplet - et l'accompagnement qui s'ensuit trop normatif - dès lors que l'on se réfère à une représentation monolithique des parcours professionnels.

\section{Des effets différenciés du passage en formation}

Cette dernière partie met les formes d'autonomie exposées dans la perspective des relations avec les acteurs de la formation et des effets du passage par l'AFPA. S'ils n'épuisent pas les configurations que l'on rencontre en formation, nous pouvons néanmoins retirer de ces résultats quelques enseignements sur l'autonomie.

L'autonomie apparaît comme une ressource pour la construction des parcours, dès lors que les situations favorisent les règles que les personnes se fixent en matière de gestion de ceux-ci. Du côté de l'AFPA, il est par exemple très attendu des stagiaires qu'ils fassent preuve de la moindre dépendance possible aux formateurs, c'est-à-dire qu'ils soient suffisamment autonomes pour s'approprier le programme de formation, en réaliser les activités et évaluer leur propre progression (Saccomanno, 2015). De même, les conseil- 
lers rencontrés en amont de la formation expriment mieux travailler à mesure que les usagers arrivent avec un projet avancé. Dans le cas inverse, en effet, la multiplication d'actions et de navettes entre programmes d'aide à la construction de projet contribue à la densification d'activités que leur groupe professionnel rencontre et regrette.

Jean-Jacques et Laetitia éprouveront peu de de difficultés à se coordonner avec les agents du système de formation lors des phases de recrutement, puis de formation, car leur engagement demeure dans la lignée de la maitrise qu'ils disent avoir de leur parcours jusque-là. Ils arrivent chacun avec un projet de métier réfléchi du point de vue de la correspondance entre l'offre de formation et les besoins du marché du travail. Leurs cas démontrent une autonomie maintenue ou renégociée suivant la règle de ne pas perdre cette liberté (par exemple, Laetitia qui souhaite s'affranchir des assignations de genre pour son orientation professionnelle).

Leur posture les amène donc à limiter les formes de dépendance aux opérateurs pour se concentrer sur leur projet post formation (qui aboutira pour chacun) ; cela les met en conformité avec les attentes du service public de l'emploi dont les agents ne sont sollicités que pour l'aspect technique de l'apprentissage et non l'accompagnement social ou l'aide à l'orientation. Ces deux formes d'autonomie favorisent ainsi un effet formation prenant la forme d'une capitalisation des ressources.

Avec l'autonomie négative, nous voyons ensuite que cette ressource peut se transformer en frein à la dynamique des parcours vers les positions souhaitées. L'autonomie est donc révélatrice de l'effet des relations sociales sur la construction des parcours. Ainsi, Samy est identifié par son manque de capacité à gérer son parcours. Dans son discours, l'autonomie est moins un objet de conquête qu'un enjeu défensif : il cherche bien moins à gagner en marge de manœuvre qu'à limiter l'effritement de sa situation. Son cas est apparu tout à fait typique de stagiaires interrogés qui escomptent de la formation qu'elle les aide à se remettre en conformité avec une normalité sociale qu'ils se représentent alors à partir des exigences des institutions qu'ils sollicitent à cet effet. Pour la majorité des institutions du marché du travail, l'autonomie (du salarié dans ses activités, du stagiaire dans son apprentissage, du demandeur d'emploi dans ses démarches...) est en tête des exigences (Dujarier, 2006, Duvoux, op. cit.). Par son attente de retour à la norme, le stagiaire s'expose à une identification négative par le manque d'autonomie que lui renverra l'institution sollicitée justement à cet effet. Ceci se ressent dans de nombreux rapprochements effectués entre l'AFPA et la formation initiale ou les organismes d'aide aux personnes en grande difficulté sociale. Une population à laquelle Samy et d'autres sont alors contraints de s'identifier malgré eux étant eux-mêmes des usagers de ces structures.

Ainsi, l'autonomie négative se donne à voir dans des systèmes relationnels paradoxaux et stigmatisant plus le manque de ressources qu'ils ne renforcent celles en présence. L'effet de la formation se trouve alors entravé par une tension entre violence intériorisée des jugements perçus et recherche d'une reconnaissance des savoirs. Cette tension émerge particulièrement lors des interactions avec n'importe quel agent en charge d'accompa- 
gner les projets. Un formateur confiera à propos de Samy : «il faut faire attention parce quill a tendance à vite se sentir en échec et alors là, il se vexe et se referme ". À deux reprises, ce dernier sera tenté de changer de filière et une fois d'abandonner (il n'ira plus en atelier durant une semaine). Il représente cette part non négligeable des publics dont le besoin de formation est validé par les prescripteurs pour des raisons d'apprentissage technique, mais aussi dans un but de resocialisation et de remise en confiance. Finalement, il abandonnera un mois avant de passer son titre et, deux ans plus tard, désormais installé dans une nouvelle région dont il nous avait évoqué le fort taux d'embauche, son parcours revient sur un cycle fait de missions intérim, chantiers au noir et périodes d'inactivité. Du point de vue de l'autonomie, son parcours semble poursuivre la pente d'un resserrement des possibilités envisagées, Samy tentant toujours de rattraper ses lacunes techniques qu'il attache à sa formation initiale. Suivant ses possibilités à être négociée, maintenue ou encore selon les blocages à son développement, l'autonomie apparaît ainsi heuristique de l'effet de l'état des marchés et des institutions sur les parcours, le cas de Samy combinant des contingences négatives à tous ces niveaux.

\section{Conclusion}

Nicolas Duvoux (op. cit.) montre combien les processus d'assignation institutionnelle des plus dominés induisent une conception normative de l'autonomie au cœur même du contrat liant les bénéficiaires aux institutions qui les accompagnent. Cette conception normative des politiques d'insertion donne lieu à des arrangements différenciés selon les profils et les parcours des administrés. Cela est confirmé par notre étude de l'autonomie au sein du système de formation, qui démontre l'impossible réduction de l'autonomie des stagiaires à un modèle unique.

Les cas retenus dans cet article sont loin d'épuiser les configurations possibles des implications de l'autonomie dans les parcours professionnels. Les trois cas travaillés ont toutefois mis au jour autant d'enjeux différenciés, éclairés par la recherche d'un surcroît d'autonomie par ces stagiaires : pour se réinventer régulièrement comme Jean-Jacques; pour renégocier son ambition professionnelle dans le cas de Laetitia ; pour tenter de revenir dans la norme du côté de Samy.

Leurs trois cas ont également souligné le caractère dynamique de l'autonomie à travers les évolutions que les personnes se font de leurs capacités à orienter leur parcours. L'autonomie apparait donc doublement comme une ressource : dans la construction des parcours et au niveau des relations sociales engagées par la mise en œuvre des projets.

La confrontation des conceptions individuelles et institutionnelles de l'autonomie révèle l'importance de compétences acquises en amont dans les parcours, afin que soit favorisée la convergence des modes d'engagement en formation avec les exigences du travail des acteurs de la formation. Ces compétences sont les multiples savoirs et pratiques mobilisables individuellement, en plus des savoirs professionnels, dans le but de correspondre 
au maximum aux attentes d'employeurs que l'on aura été en mesure de repérer soi-même. Jean-Jacques a par exemple trouvé seul sa formation et a décidé de la suivre afin d'obtenir un titre répondant aux besoins techniques d'un groupement d'employeurs auprès de qui il travaille ponctuellement et qui lui a proposé de l'embaucher. À l'inverse, le cas de Samy est celui d'un individu aux attentes décalées de ce point de vue, décalage que l'institution pointera beaucoup plus qu'elle ne lui donnera les moyens de le réduire.

L'importance accordée à l'autonomie par les règles formelles des politiques de l'emploi nous semble alors présenter le risque de durcir une appréhension normalisée de celle-ci uniquement comme capacité à se mettre soi-même en conformité avec le modèle de l'entrepreneur de soi qui gère individuellement le cours de sa carrière. Une capacité dont l'exposé des cas nous a montré qu'elle est bien plus souvent développée en amont qu'au cours de la formation. Or, ce que nous avons tenté de montrer ici, c'est que l'autonomie ne répond pas à un modèle unique de carrière souhaité, mais renvoie à une diversité de configuration de rationalités et d'effets de contextes que la notion de parcours met justement au centre de l'analyse.

\section{Bibliographie}

AFPA (2009), Rapport annuel d'activité, Montreuil, AFPA.

AFPA (2010), Rapport annuel d'activité, Montreuil, AFPA.

AFPA (2011), Rapport annuel d'activité, Montreuil, AFPA.

Appay B. (2005), La dictature du succès : le paradoxe de l'autonomie contrôlée et de la précarisation, Paris, France.

Auer P., Gazier B. (2010), L’Introuvable sécurité de l'emploi, Paris, Flammarion.

Baudelot C., Gollac M. (2003), Travailler pour être heureux ?,Le bonheur et le travail en France, Paris, Fayard.

Brinbaum Y., Guégnard C. (2012), « Parcours de formation et d'insertion des jeunes issus de l'immigration au prisme de l'orientation ", Formation Emploi, 118, pp. 61-82.

Cassagnou B. (2003), Les grandes mutations de la marine marchande française (1945-1995). Volume I, Paris, Comité pour l'histoire économique et financière de la France, coll. « GPDE «.

Corteel D., Zimmermann B. (2007), "Capacités et développement professionnel », Formation Emploi, 98, pp. 25-39.

Delory-Momberger C., Laniez G. (dirs.) (2009), Le sujet dans la cité: insertion et territoires solidaires, Nantes, Pleins Feux. 
Dujarier M.-A., 2006, "La soumission à la prescription de toute puissance ", in Durand J.-P., Le Floch M.-C. (dirs.), La question du consentement au travail. De la servitude volontaire à l'implication contrainte, Paris, L'Harmattan, « Logiques sociales «, pP. 101-110.

Duvoux N. (2009), L'autonomie des assistés, Paris, PUF, coll. « Le Lien social », 269 p

Ehrenberg A. (2010), La société du malaise, Paris, Odile Jacob.

Elias N. (1996), Du temps, Paris, Fayard.

Honneth A. (2000), La lutte pour la reconnaissance, Paris, Cerf.

Larquier G. de, Remillon D. (2008), «Assiste-t-on à une transformation uniforme des carrières professionnelles vers plus de mobilité ? ", Travail et Emploi, 113, pp. 13-30.

Longo M.E., Bourdon S., Charbonneau J., Kornig C., Mora V. (2013), « Normes sociales et imprévisibilités biographiques ", Agora débats/jeunesses, 65, 3, pp. 93-108.

Martuccelli D. (2006), Forgé par l'épreuve : l'individu dans la France contemporaine, Paris, Armand Colin.

Mauger G. (2001), "Les politiques d'insertion ", Actes de la recherche en sciences sociales, 136-137, 1, pp. 5-14.

Reynaud E. (2003), "Les règles de l'emploi », dans Terssac G. de (dir.), La théorie de la régulation sociale de Jean Daniel Reynaud. Débats et prolongements, Paris, La Découverte, coll. « Recherche», p. 147-155.

Saccomanno B. (2011), «L'intermédiation à la croisée des logiques d'acteurs : Les psychologues du travail », Formation Emploi, 114, pp. 39-54.

Saccomanno B. (2013), La formation professionnelle pour adultes : de l'éducation pour tous à la gestion individuelle des carrières, Paris, l'Harmattan.

Saccomanno B. (2015), « Modes d'engagement des stagiaires au prisme des représentations des formateurs ", Savoirs, 39, pp. 65-87.

Saccomanno B. (2016), « Négociation des ambitions et temporalités sociales », Négociations, 24.

Sainsaulieu R. (1977), L'identité au travail: les effets culturels de l'organisation, Paris, Presses de la FNSP.

Terssac G. de (1992), Autonomie dans le travail, Paris, Presses universitaires de France.

Terssac G. de, Maggi B. (1996), « Autonomie et conception », in Friedberg E., Terssac G. de (dirs.), Coopération et conception, Toulouse, Octarès, pp. 243-266.

Zimmermann B. (2011), Ce que travailler veut dire : une sociologie des capacités et des parcours professionnels, Paris, Economica. 